\title{
Receiver-Initiated vs. Short-Preamble Burst MAC Approaches for Multi-channel Wireless Sensor Networks
}

\author{
Cristina Cano, Boris Bellalta, and Miquel Oliver \\ Universitat Pompeu Fabra, \\ C/ Tànger 122-140, 08018 Barcelona, Spain \\ \{cristina.cano, boris.bellalta, miquel.oliver\}@upf .edu
}

\begin{abstract}
Wireless Sensor Networks (WSNs) are networks formed by highly constrained devices that communicate measured environmental data using low-power wireless transmissions. The increase of spectrum utilization in non-licensed bands along with the reduced power used by these nodes is expected to cause high interference problems in WSNs. Therefore, the design of new dynamic spectrum access techniques specifically tailored to these networks plays an important role for their future development. This work aims to provide insights into the performance of two well-known low energy-consuming Medium Access Control (MAC) techniques for WSNs (the receiver-initiated and the short-preamble burst), adapted to work with multiple channels. The results obtained show the best working conditions for each approach.
\end{abstract}

Keywords: WSNs, Multi-Channel, MAC, SA-MAC, Receiver-Initiated.

\section{Introduction}

Wireless Sensor Networks (WSNs) are formed by small and low-capability devices that are able to sense environmental metrics and to communicate them wirelessly to a central unit, known as a sink. The deployment of dense WSNs in large, remote and difficult-to-access areas requires keeping the size and cost of sensor nodes as low as possible. This implies that the energy, computational and memory resources of the sensor nodes are usually limited. Among the different constraints, the limitation of the energy resources is the most important one as it directly affects the network lifetime. Therefore, the design of the Medium Access Control (MAC) layer is of crucial importance because it controls the most energy consuming component of a sensor node: the transceiver. By putting the transceiver in duty cycle mode, i.e., sleeping and periodically waking up, the energy consumption can be reduced. However, a mechanism is needed to coordinate a receiver and a transmitter so as to be awake at the same instant, also known as rendezvous. Among the different techniques to ensure a rendezvous, asynchronous MAC protocols and particularly preamble sampling techniques [2] provide extremely low energy consumption at low loads (the common situation in WSNs), have a low complexity and a lack of synchronization requirements. 
Sensor nodes are normally configured to transmit at very low power [4]. This allows them to consume smaller amounts of energy while transmitting but, on the other hand, this configuration also makes them suffer from external interferences in a notable manner. It is expected that the current level of deployment of different wireless networks will make the Industrial, Scientific and Medical (ISM) band crowded in the near future [10]. Therefore, it will be necessary for a sensor device to be able to select the best portion of the spectrum to communicate with its neighbours, thus increasing the probability of a correct reception, reducing the delay and maintaining a low energy consumption per successfully sent packet. When there are multiple channels available, the rendezvous problem becomes more complicated. In that case, apart from coordinating the nodes to be awake at the same time, they also need to agree on the channel to use.

In this work, the adaptations to multi-channel operation of two well-known MAC approaches for rendezvous are evaluated and the best working conditions for each approach are obtained. These first results can be useful for the further study and design of these protocols when multiple channels are available and sensor nodes select one in which to transmit or listen for data.

This article is organized as follows. In Section 2 the existing asynchronous protocols for multi-channel operation are described. Then, in Section 3 the multichannel techniques evaluated in this article are presented. After that, an analytical derivation of these mechanisms is explained in Section 4 The description of the results obtained is presented in Section 5. Finally, some conclusions and future research directions are outlined.

\section{Related Work}

In common energy-constrained WSNs, the sensor nodes are sleeping and waking up according to their duty cycle. Therefore, a mechanism is needed to coordinate a receiver and a transmitter to communicate, since both have to be awake at the same instant. Techniques to achieve this rendezvous can be divided into three different categories: TDMA-like approaches, protocols with common active periods and asynchronous approaches [3]. Among these, asynchronous MAC protocols and particularly preamble sampling techniques consume extremely low energy when the network load is low, have a low complexity and no synchronization requirements.

Using preamble sampling, sensor nodes sample the channel periodically to detect activity and they remain awake if activity is detected. Thus, if a sensor node has something to transmit, it sends a long preamble before the data in order to wake up the receiver 7 . There are several extensions of this technique, one of the best known is the division of the long preamble into a short-preamble burst [2. By dividing the long preamble into short packets, some useful information can be included, like, for instance, the time at which the data transmission will start, allowing the receiver to go to sleep. Receiver-initiated approaches [8] follow a similar idea to the one used in preamble sampling. However, in this case, when a sensor node wants to transmit something, it wakes up and listens 
to the channel. Sensor nodes wake up periodically and send a beacon message indicating they are awake. After receiving the beacon message, the sender can start the transmission.

When there are multiple channels to use, the rendezvous problem becomes more challenging. In that case, apart from coordinating the nodes to be awake at the same time, they also need to agree on the channel to use. In this work, the attention has been placed on asynchronous approaches since, as previously stated, they provide features that are especially beneficial to WSNs.

OMA [5] and ARM [6] protocols are two asynchronous MAC protocols for multi-channel operation. While OMA is based on the transmission of a burst of short preambles, ARM is based on the receiver-initiated approach. In these protocols, the rendezvous is made in a common and fixed control channel, then the data transmission is done in the channel both receiver and transmitter agree to use.

In contrast, SA-MAC [1, that is based on the transmission of a burst of short preambles, does not use a common control channel to perform the rendezvous. When a node wakes up it samples all channels trying to find a short preamble of the receiver. The short-preamble burst should take into account, apart from the sleep time of the sensor nodes, the time to sample all the available channels. Not using a common control channel makes the protocol more robust against interference problems at the cost of a higher overhead.

Similarly, the EM-MAC 9] protocol ensures the rendezvous between receiver and transmitter in an asynchronous manner and without the use of a common control channel. The difference is that this protocol is based on the receiverinitiated technique adapted to multi-channel operation and combined with a prediction of the channel and wake-up time of the receiver based on pseudorandom numbers. This mechanism allows the transmitter to wake up right before the receiver beacon in the selected channel. As a drawback, each node must store information of each neighbour and extra overhead has to be included in messages to perform the prediction.

In this work, the receiver-initiated technique presented in [8] is adapted to multi-channel operation by making the transmitter sample all channels to find a burst of beacons of the receiver. On the other side, the transmitter-initiated technique defined in the SA-MAC protocol is adopted. The comparison of both techniques gives insights into the best working conditions for both approaches when multiple channels are available.

\section{Multi-channel Receiver and Transmitter-Initiated MACs}

In SA-MAC 1, the rendezvous based on the transmission of a short-preamble burst along with the periodic channel sampling of the receiver (similar to the single-channel technique defined in [2]) is adapted to multi-channel operation. An example of the basic functionality of this protocol with 4 channels is shown in Fig. 1. It can be observed that, when a node wants to transmit a message it 
starts transmitting a burst of short preambles in the selected channel and after that, it sends the data. The receiver, on the contrary, wakes up periodically and samples all channels. If the receiver detects activity in one channel, it remains awake to receive a complete short preamble, which includes the time at which the data transmission will start. This allows the receiver to go to sleep and wake up right before the transmission of the data message.

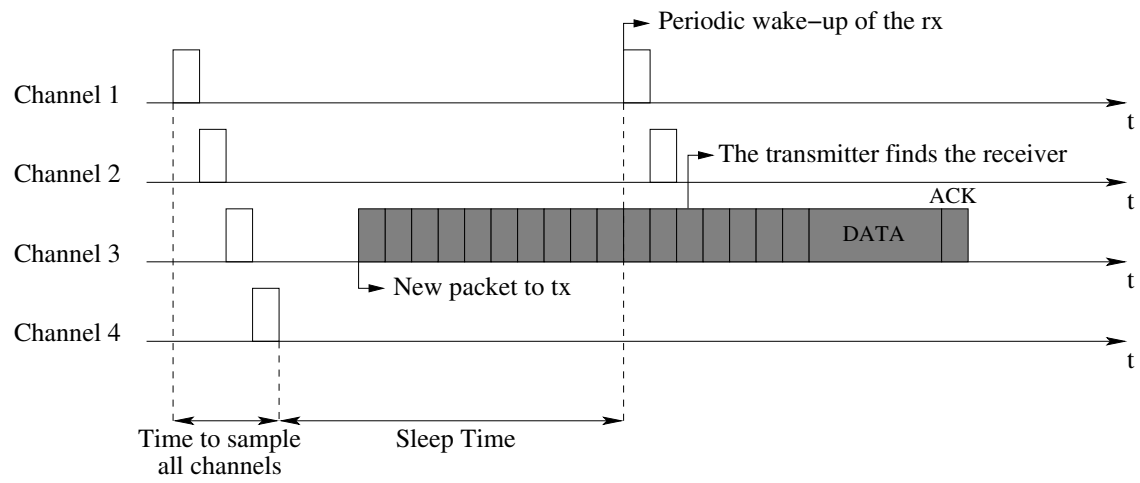

Fig. 1. Example of the short-preamble burst approach functionality with 4 channels

Observe that the rendezvous would also be possible by making the sender switch among channels and sending a short preamble on each. The receiver could then stay listening in one channel until the short preamble is received. The transmitter can either wait in each channel for an early-ACK [2] or announce in each preamble the channel in which the message will be sent. Both approaches require more energy waste and delay if compared to SA-MAC. The first case requires the transmitter to listen on each channel and the second one requires extra channel switches.

Based on the fact that modern radios (like the CC2420 transceiver 44) consume less energy in transmitting than in receiving mode, the adaptation of receiver-initiated approaches to multi-channel operation seems to be a good option, especially when the network load is low (as it typically occurs in usual WSN applications). The functionality of the receiver-initiated protocol studied in this work is shown in Fig. 2 for the case in which again 4 channels are available. Each node wakes up periodically and sends a burst (equal to the number of channels) of beacons to notify potential transmitters it is awake and ready to receive data. As previously discussed, this behaviour is expected to consume less energy than waking up to sample all channels in the cases in which the energy to receive is higher than the energy required to transmit. Then, when a node wants to transmit a packet, it sequentially and periodically scans all channels to find a beacon of the intended receiver. After the reception of a complete beacon, the transmitter goes to sleep and wakes up after the beacon burst to send the data message (assuming the network load to be low, no back-off has been considered). 
Observe that the transmitter should sample the channels during the sleep time plus twice the beacon burst duration to ensure the rendezvous.

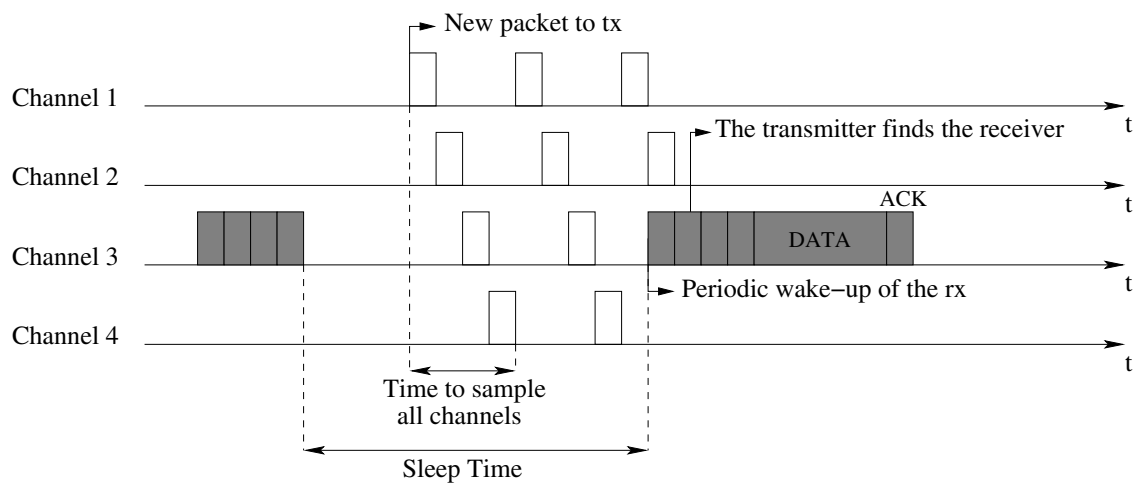

Fig. 2. Example of the receiver-initiated approach functionality with 4 channels

Observe also that the rendezvous could alternatively be done in this case by having the receiver switching among channels with the transmitter waiting for the reception of a beacon in a given channel. In this design, the receiver should either stay awake in every channel after the transmission of the beacon waiting for data or announce the channel in which it will be listening for data. Again, these alternatives consume more energy or entail more delay than the one previously described.

\section{Analytical Derivation of Energy Consumption}

To analyze and compare the performance of both techniques with multi-channel operation we conduct an analysis of the energy consumption. The energy consumed by each sensor during a time $T$ has been calculated. Assuming that each node generates packets at an average rate of $\lambda$ packets/s, the number of generated messages during $T$ is $N=\lambda \cdot T$. Then, the goal is to find the total energy spent $(e)$ as the sum of the energy required to transmit these messages $\left(e_{\mathrm{tx}}\right)$, the energy spent receiving messages from other nodes $\left(e_{\mathrm{rx}}\right)$ and the energy wasted during the duty cycle $\left(e_{\mathrm{dc}}\right)$, i.e., sleeping and periodically waking up to either sample all channels or to send the burst of beacons: $e=e_{\mathrm{tx}}+e_{\mathrm{rx}}+e_{\mathrm{dc}}$.

The analytical model presented in this work considers the following assumptions:

1. It is assumed that, as occurs on common platforms like the MicaZ [4] nodes, each node is equipped with only one radio that can be tuned to work in different channels, one at a time. 
2. The traffic load within the WSN is low enough to consider the collision probability negligible. This is a common assumption in WSN analytical models and is also used in [1].

3. A node sends and receives the same amount of messages and it is assumed that there is no overhearing, i.e., nodes only receive the packets addressed to them.

4. Ideal or random (as done in [5] and [6]) channel selection is assumed. Since the purpose is to evaluate the behaviour of the rendezvous mechanisms, the channel selection strategy does not affect the comparison.

\subsection{Short-Preamble Burst Approach}

Let $t_{\text {speriod }}$ be the sampling period of a sensor node, i.e., the time between two channel samples, and $t_{\mathrm{s}}$ the time required to sample one channel. Therefore, the sleep time $\left(t_{\text {sleep }}\right)$ can be computed as shown in Eq. 1, where $n_{\text {ch }}$ is the number of available channels.

$$
t_{\text {sleep }}=t_{\text {speriod }}-\left(n_{\mathrm{ch}} \cdot t_{\mathrm{s}}\right)
$$

Then, the time to transmit a message (Eq. 2) can be calculated as the sum of the time to transmit the short-preamble burst (it has to be transmitted during the $t_{\text {speriod }}$ to ensure a correct rendezvous $)$, the time to transmit the data $\left(t_{\text {data }}\right)$ packet and the time to receive the ACK $\left(t_{\text {ack }}\right)$.

$$
T_{\mathrm{tx}}=t_{\mathrm{speriod}}+t_{\mathrm{data}}+t_{\mathrm{ack}}
$$

The energy consumption spent to transmit a message can be obtained by multiplying the time spent in each mode by the power drawn in the specific mode ( $P_{i}$, where $i$ can be transmit, receive or sleep mode) as shown in Eq. 3.

$$
E_{\mathrm{tx}}=P_{\mathrm{tx}} \cdot\left(t_{\text {speriod }}+t_{\mathrm{data}}\right)+P_{\mathrm{rx}} \cdot t_{\mathrm{ack}}
$$

The total time and energy consumption to transmit all messages generated during a time $T$ is: $t_{\mathrm{tx}}=N \cdot T_{\mathrm{tx}}$ and $e_{\mathrm{tx}}=N \cdot E_{\mathrm{tx}}$.

Similarly, we can compute the time (Eq. 4) and energy spent to receive a message (Eq. 5) assuming that each node will receive the same amount of messages than it generates. In this case, it has to be considered that the receiver will find the transmitter on average in $\left(n_{\mathrm{ch}} \cdot t_{\mathrm{s}}\right) / 2$ and that it will only receive on average 1.5 short preamble messages [1] of duration $t_{\text {short }-\mathrm{p}}$.

$$
\begin{gathered}
T_{\mathrm{rx}}=\frac{\left(n_{\mathrm{ch}} \cdot t_{\mathrm{s}}\right)}{2}+\left(1.5 \cdot t_{\text {short }-\mathrm{p}}\right)+t_{\mathrm{data}}+t_{\mathrm{ack}} \\
E_{\mathrm{rx}}=P_{\mathrm{rx}} \cdot\left(\frac{\left(n_{\mathrm{ch}} \cdot t_{\mathrm{s}}\right)}{2}+\left(1.5 \cdot t_{\text {short }-\mathrm{p}}\right)+t_{\mathrm{data}}\right)+\left(P_{\mathrm{tx}} \cdot t_{\mathrm{ack}}\right)
\end{gathered}
$$

The total time receiving and the total energy spent to receive $N$ messages is also: $t_{\mathrm{rx}}=N \cdot T_{\mathrm{rx}}$ and $e_{\mathrm{rx}}=N \cdot E_{\mathrm{rx}}$, respectively. 
The rest of the time $\left(t_{\mathrm{dc}}=T-t_{\mathrm{tx}}-t_{\mathrm{rx}}\right)$, a node will be in duty cycle, waking up to sample all channels and sleeping. The energy spent in this operation is shown in Eq. 6.

$$
e_{\mathrm{dc}}=t_{\mathrm{dc}} \cdot \frac{\left(P_{\mathrm{rx}} \cdot\left(n_{\mathrm{ch}} \cdot t_{\mathrm{s}}\right)\right)+\left(P_{\mathrm{sleep}} \cdot t_{\mathrm{sleep}}\right)}{\left(n_{\mathrm{ch}} \cdot t_{\mathrm{s}}\right)+t_{\mathrm{sleep}}}
$$

\subsection{Receiver-Initiated Approach}

In the receiver-initiated approach, the sleep time can be defined in a similar way. However, it has to be considered that each node sends a total of $n_{\mathrm{ch}}$ beacons each time it wakes up:

$$
t_{\text {sleep }}=t_{\text {speriod }}-\left(n_{\mathrm{ch}} \cdot t_{\text {beacon }}\right)
$$

To compute the time and energy required to transmit a message, it has been assumed that a transmitter samples the channels during $\left(t_{\text {sleep }}+\left(2 \cdot n_{\text {ch }} \cdot t_{\text {beacon }}\right)\right) / 2$ $\mathrm{s}$, on average, to find a receiver and that it will receive, also on average, 1.5 beacons. The expression to compute that time is described in Eq. 8 and the corresponding energy consumption is shown in Eq. 9.

$$
\begin{gathered}
T_{\mathrm{tx}}=\frac{t_{\text {sleep }}+\left(2 \cdot n_{\mathrm{ch}} \cdot t_{\text {beacon }}\right)}{2}+\left(1.5 \cdot t_{\text {beacon }}\right)+t_{\text {data }}+t_{\text {ack }} \\
E_{\mathrm{tx}}=P_{\mathrm{rx}} \cdot\left(\frac{t_{\text {sleep }}+\left(2 \cdot n_{\mathrm{ch}} \cdot t_{\text {beacon }}\right)}{2}+\left(1.5 \cdot t_{\text {beacon }}\right)\right)+\left(P_{\mathrm{tx}} \cdot t_{\text {data }}\right)+\left(P_{\mathrm{rx}} \cdot t_{\mathrm{ack}}\right)
\end{gathered}
$$

The time and energy to receive a message is computed considering that a node sends $n_{\text {ch }}$ beacons, receives the data and sends the ACK as shown in Eq. 10 and Eq. 11 respectively.

$$
\begin{gathered}
T_{\mathrm{rx}}=\left(n_{\mathrm{ch}} \cdot t_{\text {beacon }}\right)+t_{\text {data }}+t_{\mathrm{ack}} \\
E_{\mathrm{rx}}=\left(P_{\mathrm{tx}} \cdot n_{\mathrm{ch}} \cdot t_{\text {beacon }}\right)+\left(P_{\mathrm{rx}} \cdot t_{\mathrm{data}}\right)+\left(P_{\mathrm{tx}} \cdot t_{\mathrm{ack}}\right)
\end{gathered}
$$

The energy spent in duty cycle has to take into account that when a node wakes up it sends $n_{\text {ch }}$ beacons (Eq. [12):

$$
e_{\mathrm{dc}}=t_{\mathrm{dc}} \cdot \frac{\left(P_{\mathrm{tx}} \cdot\left(n_{\mathrm{ch}} \cdot t_{\text {beacon }}\right)\right)+\left(P_{\text {sleep }} \cdot t_{\mathrm{sleep}}\right)}{\left(n_{\mathrm{ch}} \cdot t_{\text {beacon }}\right)+t_{\text {sleep }}}
$$

\section{Numerical Evaluation}

Using the analytical models described in Section 4, we now conduct a numerical evaluation. Results varying the packet transmission rate with $n_{\mathrm{ch}}$ set to $5,10,15$ 
Table 1. Evaluation Parameters

\begin{tabular}{|c|c||c|c|}
\hline Parameter & Value & Parameter & Value \\
\hline$t_{\text {data }}$ & $3.2 \mathrm{~ms}$ & $t_{\text {ack }}$ & $0.32 \mathrm{~ms}$ \\
\hline$t_{\text {beacon }}=t_{\text {short }-\mathrm{p}}$ & $15.8 \mathrm{~ms}$ & $t_{\mathrm{s}}$ & $15.8 \mathrm{~ms}$ \\
\hline Time $(T)$ & $1000 \mathrm{~s}$ & $P_{\mathrm{tx}}$ & $46.5 \mathrm{~mW}$ \\
\hline$P_{\mathrm{rx}}$ & $58.9 \mathrm{~mW}$ & $P_{\text {sleep }}$ & $3.6 \mathrm{~mW}$ \\
\hline
\end{tabular}

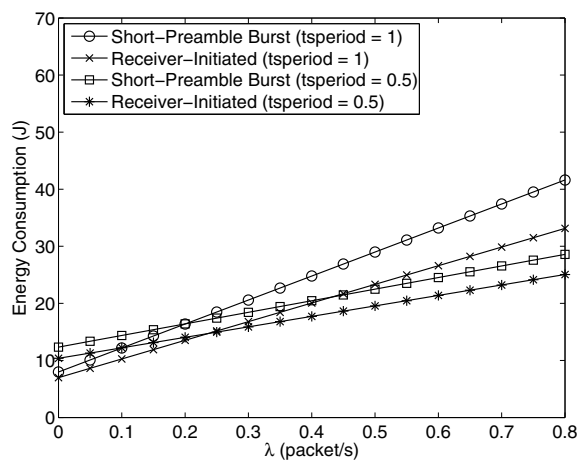

(a) $n_{\mathrm{ch}}=5$

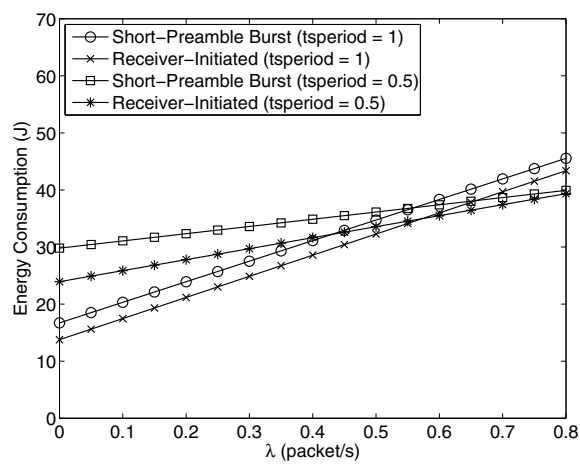

(c) $n_{\mathrm{ch}}=15$

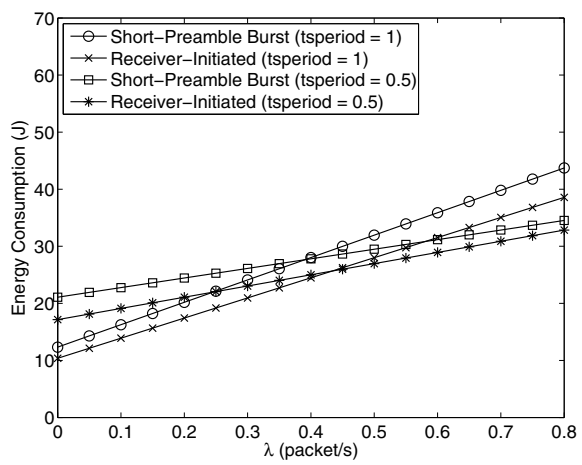

(b) $n_{\mathrm{ch}}=10$

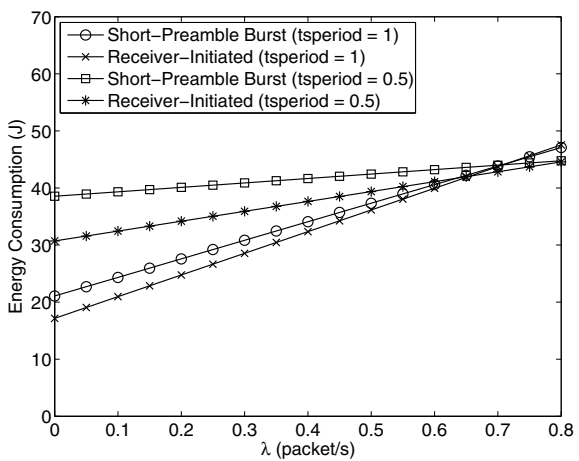

(d) $n_{\text {ch }}=20$

Fig. 3. Energy Consumption with different number of channels and traffic loads

and 20 are shown in Fig. 3. The default parameters used for the evaluation were measured on the TelosB platform by Ansari et al. in [1] and are shown in Table 1

In Fig. 3, it can be observed that when the traffic of the network is low, the receiver-initiated approach consumes less energy than the short-preamble burst technique, independently of the $t_{\text {speriod }}$ and the number of channels. In the short-preamble burst approach each node periodically wakes up to sample 
the channel during $n_{\mathrm{ch}} \cdot t_{\mathrm{s}}$. In contrast, in the receiver-initiated approach each node periodically sends beacons during $n_{\mathrm{ch}} \cdot t_{\text {beacon}}$. Given that $t_{\mathrm{s}}$ has been considered equal to $t_{\text {beacon }}$ and that it has been assumed that the energy to receive is higher than the energy required to transmit, the energy consumption for the receiver-initiated technique at low loads will always be lower than the one spent in the short-preamble burst case, independently of the number of available channels. However, as the number of channels increases, the difference is more notable.

On the other hand, note that for higher network loads the receiver-initiated approach also consumes less energy than the short-preamble burst approach in most of the cases evaluated. The difference is especially notable when the number of channels is small and $t_{\text {sleep }}$ is long. This difference becomes smaller when the number of channels increases and $t_{\text {sleep }}$ is reduced. The short-preamble burst can even show a slightly lower energy consumption than the receiver-initiated approach as depicted in Fig. $3(\mathrm{~d})$. For each message to transmit, the short-preamble burst technique sends a burst of preambles during $t_{\text {sleep }}+\left(n_{\mathrm{ch}} \cdot t_{\text {short-p }}\right)$, independently of when the rendezvous occurs. In contrast, in the receiver-initiated approach, the transmitter stops listening the channels when it receives a beacon. This happens on average in a period of $\left(t_{\text {sleep }}+\left(2 \cdot n_{\mathrm{ch}} \cdot t_{\text {beacon }}\right)\right) / 2$. Therefore, when both the number of channels increases and $t_{\text {sleep }}$ is reduced, the energy consumption of the short-preamble burst mechanism can become smaller than the consumption of the receiver-initiated approach.

\section{Conclusions and Future Work}

The capability of coordinating low-capable sensor nodes working at different frequency bands is still a research challenge. In this case, multi-channel communication has been considered. However, it will provide higher benefits and also higher challenges to select variable portions of the spectrum in which to transmit based on the conditions observed. Sensor nodes will, in this scenario, have to coordinate themselves to aggree on the portion of the spectrum to use.

In this work, the receiver-initiated rendezvous approach has been adapted to work with multiple channels and compared to the multi-channel approach based on the transmission of a short-preamble burst. Results have shown that the energy consumed by the receiver-initiated approach is smaller than the energy spent by the short-preamble burst technique in scenarios with low traffic load and also in cases with high traffic load, reduced number of channels and high sleep time.

This work aims to provide the first comparison of both techniques for multichannel operation. However, a complete evaluation in multi-hop and large scenarios is required and has been left as a future work. Moreover, new approaches need to be defined in order to reduce the unnecessary energy spent to ensure the rendezvous in a multi-channel WSNs without the use of a common control channel. 
Acknowledgments. This work has been partially supported by the Spanish Government under projects TEC2008-06055 (Plan Nacional I+D) and CSD200800010 (Consolider-Ingenio Program), and by the Catalan Government (SGR2009 \#00617).

\section{References}

1. Ansari, J., Ang, T., Mahonen, P.: Spectrum agile medium access control protocol for wireless sensor networks. In: IEEE Communications Society Conference on Sensor Mesh and Ad Hoc Communications and Networks (SECON), pp. 1-9 (2010)

2. Buettner, M., Yee, G., Anderson, E., Han, R.: X-MAC: A Short Preamble MAC Protocol for Duty-cycled Wireless Sensor Networks. In: Proceedings of the 4th International Conference on Embedded Networked Sensor Systems (Sensys 2006), pp. 307-320 (2006)

3. Cano, C., Bellalta, B., Sfairopoulou, A., Oliver, M.: Low energy operation in wsns: A survey of preamble sampling mac protocols. Computer Networks (2011)

4. Chipcon: CC2420. 2.4 GHz IEEE 802.15.4 / ZigBee-ready RF Transceiver

5. Li, J., Zhang, D., Guo, L.: OMA: a multi-channel mac protocol with opportunistic media access in wireless sensor networks. In: International Conference on Mobile Ad-hoc and Sensor Networks (MSN), pp. 7-13 (2011)

6. Li, J., Zhang, D., Guo, L., Ji, S., Li, Y.: ARM: an asynchronous receiver-initiated multi-channel mac protocol with duty cycling for wsns. In: Performance Computing and Communications Conference, IPCCC (2010)

7. Polastre, J., Hill, J., Culler, D.: Versatile Low Power Media Access for Wireless Sensor Networks. In: Proceedings of the 2nd International Conference on Embedded Networked Sensor Systems, SenSys 2004 (2004)

8. Sun, Y., Gurewitz, O., Johnson, D.: RI-MAC: A Receiver-initiated Asynchronous Duty Cycle MAC Protocol for Dynamic Traffic Loads in Wireless Sensor Networks. In: Proceedings of the 6th ACM Conference on Embedded Network Sensor Systems (Sensys 2008), pp. 1-14 (2008)

9. Tang, L., Sun, Y., Gurewitz, O., Johnson, D.: EM-MAC: A dynamic multichannel energy-efficient mac protocol for wireless sensor networks. In: ACM International Symposium on Mobile Ad Hoc Networking and Computing, MobiHoc (2011)

10. Zhou, G., Stankovic, J., Son, S.: Crowded Spectrum in Wireless Sensor Networks. IEEE EmNets (2006) 